\title{
Lyme Borreliosis with Scalp Eschar Mimicking Rickettsial Infection, Austria
}

\author{
Mateusz Markowicz, Anna-Margarita Schötta, Michiel Wijnveld, Gerold Stanek
}

We report on a patient in Austria with scalp eschar and neck lymphadenopathy. Rickettsial etiology was excluded by culture, PCR, and serologic tests. Borrelia afzelii was identified from the eschar swab by PCR. Lyme borreliosis can mimic rickettsiosis; appropriate tests should be included in the diagnostic workup of patients with eschars.

Calp eschar and neck lymphadenopathy (SENSLAT) is frequently caused by Rickettsia slovaca and $R$. raoultii. Reports on other etiologic agents have expanded the knowledge about the cause of this clinical entity $(1,2)$. In some cases the causative agents remain undetermined. We report a case of SENLAT in a patient from Austria with serologic and molecular evidence for infection with Borrelia burgdorferi sensu lato (s.l.).

\section{The Patient}

A 61-year-old woman was referred to the outpatient department of the Institute for Hygiene and Applied Immunology, Medical University of Vienna (Vienna, Austria), because of fatigue, muscle pain, and elevated body temperature $\left(37.7^{\circ} \mathrm{C}\right)$ of 1 week's duration. Before the onset of symptoms she reported having a tick bite on her head, and she noticed a palpable crust at the site of the bite. The patient was healthy, aside from having arterial hypertension and hypothyroidism, and she reported having contact with ticks and horses. She stated that ticks were highly abundant in her garden and frequently infested the horses. The physical examination revealed a crusty lesion measuring $0.5 \mathrm{~cm}$, compatible with an eschar (Figure); lymph nodes in the neck were enlarged. The clinical picture and the history of tick exposure suggested a rickettsial infection.

Basic laboratory tests showed cell counts and liver function parameters within reference ranges,

Author affiliation: Medical University of Vienna, Vienna, Austria

DOI: https://doi.org/10.3201/eid2609.191256 elevated lactate dehydrogenase (341 U/L, reference $<247 \mathrm{U} / \mathrm{L}$ ), and moderately elevated C-reactive protein $(0.59 \mathrm{mg} / \mathrm{dL}$, reference $<0.5 \mathrm{mg} / \mathrm{dL})$. Serologic tests for tickborne diseases using Anti-Borrelia plus VlsE ELISA for IgG and Anti-Borrelia ELISA for IgM (Euroimmun, https://www.euroimmun.com), Rickettsia IFA IgG (Focus Diagnostics, https://www.focusdx.com), and Weil-Felix agglutination assay (DiaMondial, http://www.diamondial.com) all showed results below the cutoff levels and were interpreted as negative. In addition, we obtained material from the scalp eschar for PCR and culture by removing the crust with sterile tweezers. We used parts of the crust for testing, together with a swab taken directly from the skin underneath the crust. We isolated DNA from the swab and crust parts using the PeqGOLD Tissue DNA Mini Kit (Peqlab, https://de.vwr.com/cms/ life_science), according to the manufacturer's instructions. We used real-time PCR to target the gltA gene of Rickettsia spp. (3). We also attempted to culture Rickettsia spp. as previously described (4). We placed tissue from the eschar site directly into a tick cell-culture vial containing a layer of BME/CTVM2 cells. The cell line was provided by Lesley Bell-Sakyi, The Tick Cell Biobank, Institute of Infection and Global Health, University of Liverpool (Liverpool, UK), and cultivated as described previously $(5,6)$. We supplemented the L-15 medium (Leibovitz's L-15 medium; GIBCO, Thermo Fisher Scientific, https:/ / www.thermofisher. com) with an antibiotic-antimycotic mixture (GIB$\mathrm{CO})$ to prevent unwanted contamination of bacterial or fungal origin. We prepared cytocentrifuge smears weekly from culture samples $(\approx 100 \mu \mathrm{L})$ and stained them using the Shandon Kwik-Diff kit (Thermo Fisher Scientific).

The rickettsial PCR was negative, and cultivation was not successful. To confirm the negative culture, we took samples containing supernatant and cells after 2 months' incubation and tested them with the aforementioned real-time PCRs for B. burgdorferi s.l. 
and Rickettsia spp. but also received negative results. At the patient's initial admission, we started treatment with doxycycline $200 \mathrm{mg}$ for 10 days. and the patient reported a rapid improvement of her symptoms.

Three weeks after we began treatment, we repeated the serologic tests for the respective tickborne pathogens and measured high concentrations of antibodies against $B$. burgdorferi s.l.: IgG $44 \mathrm{U} / \mathrm{mL}$ (positive cutoff $>22 \mathrm{U} / \mathrm{mL}$ ) and $\operatorname{IgM}>200 \mathrm{U} / \mathrm{mL}$ (positive cutoff $>22 \mathrm{U} / \mathrm{mL}$ ). We used an immunoblot (AntiBorrelia Euroline-RN-AT, Euroimmun) as a confirmatory test: for IgM, we observed strong reactivity for antigens p41, p39, and OspC; for IgG, we found strong reactivity for $\mathrm{p} 41$ and $\mathrm{OspC}$ and weak reactivity for VlsE. Levels of antibodies against Rickettsia spp. remained negative.

These findings confirmed that the patient had an infection with B. burgdorferi s.l.; therefore, we further

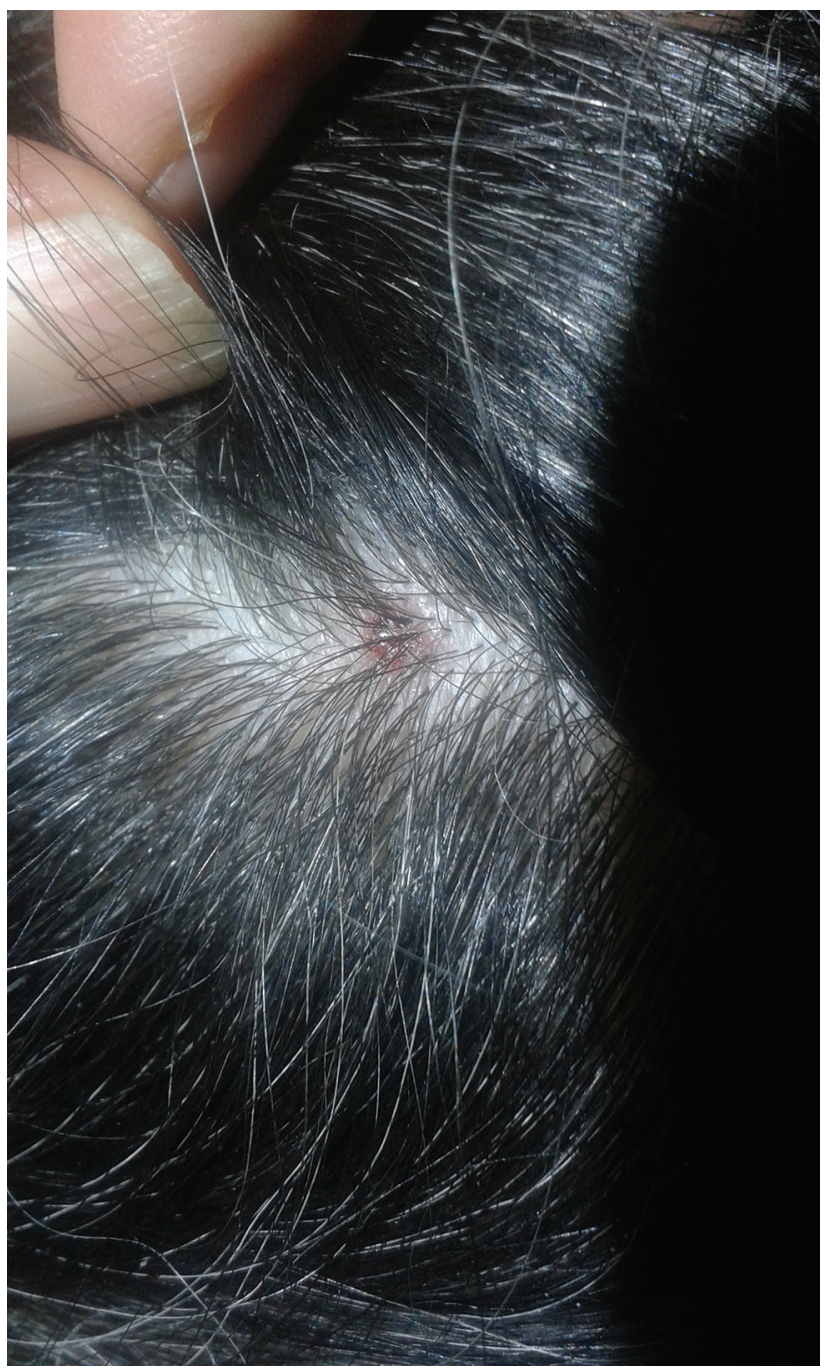

Figure. Scalp eschar of a woman in Austria who was found to be infected with Borrelia afzelii. analyzed the DNA extract from the swab to search for borrelial DNA. We used 2 real-time PCRs based on the $16 \mathrm{~S}$ rRNA gene (7) and the flagellin gene (3); the 16S PCR gave a positive result. We used a nested PCR targeting the 5S-23S intergenic spacer region $(8,9)$ for genospecies identification and purified the obtained amplicon using the QIAGEN Gel Extraction Kit (https://www.qiagen.com) before bidirectional sequencing (Microsynth, https://www.microsynth. ch). Comparison with known sequences available at the National Center for Biotechnology Information (https://blast.ncbi.nlm.nih.gov/Blast.cgi) yielded a $100 \%$ match to various B. afzelii strains.

\section{Conclusions}

The most common skin manifestation of Lyme borreliosis is solitary erythema migrans (EM) (10); multiple EM, borrelial lymphocytoma, and acrodermatitis chronica atrophicans occur less frequently. Eschar formations are local necrotic skin alterations covered by a crust and occur at the site of a tick bite after infection with tickborne rickettsial species. Enlargement of local lymph nodes, skin rash, and malaise are additional symptoms that may be observed (11). Diagnosis for patients with EM is based on the clinical picture only; no laboratory support is needed in most cases. Rickettsia spp. can be easily identified by PCR, either from skin tissue obtained from the eschar or by taking a swab from the same site for PCR testing. Serologic tests may be helpful if PCR is not available. A 2-step increase of the antibody titer in consecutive samples in an immunofluorescence assay is needed for confirmation of infection. Treatment with doxycycline is effective for both diseases.

Ixodes ricinus ticks are capable of transmitting several pathogenic species of the B. burgdorferi s.l. complex; it is the most common tick species that feeds on humans in central Europe. B. afzelii has been recognized as the most frequent genotype associated with EM in Europe. In a study in Slovenia, $89 \%$ of Borrelia species detected from skin biopsies of EM patients were B. afzelii (12). Similarly, B. afzelii was the most prevalent Borrelia species found in ticks in Austria (13). In a recent study we showed that $56 \%$ of all I. ricinus ticks positive for B. burgdorferi sl harbored B. afzelii (13). The range of tick vectors of Rickettsia spp. is much more diverse; Dermacentor reticulatus and $D$. marginatus ticks are known to transmit $R$. slovaca and $R$. raoultii (11). We asked the patient to collect ticks from the area where she lives for identification of the tick species abundant there. The morphological examination of ticks collected from vegetation revealed only the presence of 
I. ricinus ticks; thus, it is unlikely that a species of Dermacentor tick transmitted the infection.

Identification of B. afzelii from the eschar of our patient was unexpected. Ni et al. have shown that eschar formations can occur after borrelial infection in patients with concomitant EM (14); however, it is not known whether those patients were also infected with Rickettsia spp. A molecular identification of B. burgdorferi s.l. in a tick from a patient with SENLAT has been reported (2), and our case study provides clear evidence that the spirochete can be detected directly in the scalp eschar. Moreover, we are not aware of any other report of molecular identification of $B$. afzelii from a skin swab and superficial skin material. Inclusion of B. burgdorferi in the differential diagnosis of patients with SENLAT appears justified, particularly if testing for Rickettsia spp. is not successful.

\section{About the Author}

Dr. Markowicz is a medical specialist in general medicine and hygiene and microbiology at the Medical University of Vienna, Vienna, Austria. His primary research interests are Lyme borreliosis and other bacterial tickborne diseases. He is also an unpaid member of the Executive Committee of ESGBOR, the ESCMID Study Group on Lyme Borreliosis.

\section{References}

1. Angelakis E, Pulcini C, Waton J, Imbert P, Socolovschi C, Edouard S, et al. Scalp eschar and neck lymphadenopathy caused by Bartonella henselae after tick bite. Clin Infect Dis. 2010;50:549-51. https://doi.org/10.1086/650172

2. Dubourg G, Socolovschi C, Del Giudice P, Fournier PE, Raoult D. Scalp eschar and neck lymphadenopathy after tick bite: an emerging syndrome with multiple causes. Eur J Clin Microbiol Infect Dis. 2014;33:1449-56. https://doi.org/10.1007/s10096-014-2090-2

3. Leschnik M, Khanakah G, Duscher G, Wille-Piazzai W, Hörweg C, Joachim A, et al. Species, developmental stage and infection with microbial pathogens of engorged ticks removed from dogs and questing ticks. Med Vet Entomol. 2012;4:440-6. https:/ / doi.org/10.1111/ j.1365-2915.2012.01036.x

4. Wijnveld M, Schötta A-M, Pintér A, Stockinger H, Stanek G. Novel Rickettsia raoultii strain isolated and propagated from Austrian Dermacentor reticulatus ticks. Parasit Vectors. 2016;9:567. https:// doi.org/10.1186/s13071-016-1858-x
5. Bell-Sakyi L. Ehrlichia ruminantium grows in cell lines from four ixodid tick genera. J Comp Pathol. 2004;130:285-93. https:// doi.org/10.1016/j.jcpa.2003.12.002

6. Alberdi MP, Nijhof AM, Jongejan F, Bell-Sakyi L. Tick cell culture isolation and growth of Rickettsia raoultii from Dutch Dermacentor reticulatus ticks. Ticks Tick Borne Dis. 2012;3:349-54. https:// doi.org/10.1016/j.ttbdis.2012.10.020

7. Tsao JI, Wootton JT, Bunikis J, Luna MG, Fish D, Barbour AG. An ecological approach to preventing human infection: vaccinating wild mouse reservoirs intervenes in the Lyme disease cycle. Proc Natl Acad Sci U S A. 2004;101: 18159-64. https://doi.org/10.1073/pnas.0405763102

8. Postic D, Assous MV, Grimont PA, Baranton G. Diversity of Borrelia burgdorferi sensu lato evidenced by restriction fragment length polymorphism of rrf (5S)-rrl (23S) intergenic spacer amplicons. Int J Syst Bacteriol. 1994;44: 743-52. https:/ / doi.org/10.1099/00207713-44-4-743

9. Wilhelmsson P, Fryland L, Börjesson S, Nordgren J, Bergström S, Ernerudh J, et al. Prevalence and diversity of Borrelia species in ticks that have bitten humans in Sweden. J Clin Microbiol. 2010;48:4169-76. https:/ / doi.org/10.1128/ JCM.01061-10

10. Stanek G, Wormser GP, Gray J, Strle F. Lyme borreliosis. Lancet. 2012;379:461-73. https:/ / doi.org/10.1016/S01406736(11)60103-7

11. Parola P, Paddock CD, Socolovschi C, Labruna MB, Mediannikov O, Kernif T, et al. Update on tick-borne rickettsioses around the world: a geographic approach. Clin Microbiol Rev. 2013;26:657-702. https:/ / doi.org/10.1128/ CMR.00032-13

12. Ruzić-Sabljić E, Maraspin V, Lotric-Furlan S, Jurca T, Logar M, Pikelj-Pecnik A, et al. Characterization of Borrelia burgdorferi sensu lato strains isolated from human material in Slovenia. Wien Klin Wochenschr. 2002; 114:544-50.

13. Schötta AM, Wijnveld M, Stockinger H, Stanek G. Approaches for reverse line blot-based detection of microbial pathogens in Ixodes ricinus ticks collected in Austria and impact of the chosen method. Appl Environ Microbiol. 2017;83:e00489-17. https:/ / doi.org/10.1128/ AEM.00489-17

14. Ni X-B, Jia N, Jiang B-G, Sun T, Zheng Y-C, Huo Q-B, et al. Lyme borreliosis caused by diverse genospecies of Borrelia burgdorferi sensu lato in northeastern China. Clin Microbiol Infect. 2014;20:808-14. https:/ / doi.org/10.1111/14690691.12532

Address for correspondence: Mateusz Markowicz, Institute for Hygiene and Applied Immunology, Center for Pathophysiology, Infectiology and Immunology, Medical University of Vienna, Kinderspitalgasse 15, 1090 Vienna, Austria; email: mateusz.markowicz@meduniwien.ac.at 